\title{
The Burden of Hyperbilirubinemia in Critically III Patients with Hematological Malignancies: Post-hoc Analysis of a Prospective Multicenter Multinational Study
}

Magali Bisbal ( $\sim$ bisbalm@ipc.unicancer.fr)

Institut Paoli-Calmettes https://orcid.org/0000-0001-6571-0861

Michael Darmon

Hopital Saint Louis

Colombe Saillard

Institut Paoli-Calmettes

Vincent Mallet

Hopital Cochin

Charlotte Mouliade

Hopital Cochin

Virginie Lemiale

Hopital Saint Louis

Frederic Pene

Hopital Cochin

Achille Kouatchet

Centre Hospitalier Universitaire d'Angers

Alexandre Demoule

Hopital Universitaire Pitie Salpetriere

Francois Vincent

Hopital Avicenne

Martine Nyunga

Centre Hospitalier de Roubaix

Fabrice Bruneel

Centre Hospitalier de Versailles

Christine Lebert

Centre Hospitalier Departemental Vendee

Anne Renault

CHRU de Brest

Anne-Pascale Meert

Institut Jules Bordet 


\section{Dominique Benoit}

Universiteit Gent

\section{Rebecca Hamidfar}

Centre Hospitalier Universitaire Grenoble Alpes Hopital Michallon

\section{Merce Jourdain}

"Centre Hospitalier Regional Universitaire de Lille"

\section{Elie Azoulay}

Hopital Saint Louis

Djamel Mokart

Institut Paoli-Calmettes

\section{Research}

Keywords: Hyperbilirubinemia, outcome, critically ill patient, hematological malignancy, liver biopsy

Posted Date: March 4th, 2020

DOl: https://doi.org/10.21203/rs.3.rs-15794/v1

License: (c) (i) This work is licensed under a Creative Commons Attribution 4.0 International License. Read Full License 


\section{Abstract}

\section{Background}

The evidence on the clinical significance of hyperbilirubinemia (HB) in critically ill patients with hematological malignancies is scarce. We therefore studied its burden in a 2010-2011 Franco-Belgian multicenter prospective study designed to evaluate the prognosis of these patients.

\section{Patients and methods}

The cohort comprised 893 patients from 17 centers, 61\% men, with a median (interquartile range) age of 60 (49 - 70) years, and preferentially with underlying non-Hodgkin lymphoma (32\%) or acute myeloid leukemia (27\%). HB was defined as a total serum bilirubin $\geq 33 \mu \mathrm{mol} / \mathrm{L}$ at intensive care unit (ICU) admission. Our main goal was to evaluate the relationship between HB and outcome of critically ill hematological patients. Causes and management of HB in the ICU were analyzed as secondary end points.

\section{Results}

HB concerned 185 (21\%) patients. Cyclosporine and antimicrobial treatments, ascites and cirrhosis, acute kidney injury, neutropenia, and myeloma (adjusted odd ratio [aOR] 0.38, $\mathrm{p}=0.006$ ) were risk factors. Hospital mortality was $56.3 \%$ and $36.3 \%$ in patients with and without $\mathrm{HB}$, respectively $(\mathrm{p}<0.0001$ with the log-rank test). Adjusted for severity of illness, the adjusted odds ratio ( $95 \%$ confidence interval) of HB for in-hospital mortality was $1.86(1.28,2.72)$. HB was overlooked by the ICU team for $92(53 \%)$ patients. Overwise, liver workups for HB led to treatment modifications in $32(40 \%)$ patients, including chemotherapy for cancer progression that was associated with reduced mortality with an adjusted odds ratio of $0.23,(p=0.02)$.

\section{Conclusion}

$\mathrm{HB}$ is associated with outcome of critically ill hematological adult patients and should be systematically explored and treated.

\section{Introduction}

Liver abnormalities are common in critically ill patients with hematological malignancies and is reported after hematopoietic stem cell transplantation (HSCT) $(1,2)$, targeted therapies, immunoconjugate antibodies and immunotherapies (3-5). Causes are frequently multifactorial, including drug-induced liver injury (DILI) (6-8), transfusional iron overload (9), infections (10), sepsis (11), prolonged parenteral nutrition (12), underlying hepatic disease (13) and cancer-related complications such as tumoral infiltration (14), hepatic graft-versus-host disease (GvHD) after allogenic HSCT (allo-HSCT) (15), sinusoidal obstruction syndrome (SOS) $(16,17)$, tumor lysis syndrome (18) and haemophagocytic syndrome (19). 
Diagnosis of liver injury in critically ill hematological patients is challenging as clinical, biological and radiological findings are, in general, nonspecific, and because of the absence of systematic guidelines, including on the use of liver biopsies. Total serum bilirubin level, a biomarker of liver function, has been incorporated in several organ dysfunction scores $(20,21)$ to grade liver injury in intensive care patients. Hyperbilirubinemia (HB), defined as an increased total serum bilirubin level $\geq 68 \mu \mathrm{mol} / \mathrm{L}$, has been associated with a doubled risk of mortality in a large cohort allo-HSCT patients $(22,23)$. In pediatric patients, a total serum bilirubin $\geq 33 \mu \mathrm{mol} / \mathrm{L}$ one month after allo-HSCT has been associated with higher non-relapse mortality (24). HB seems more associated with mortality than hepatocellular injury, defined as elevated aminotransferase levels without HB in hematological patients (25).

Overall, the level of evidence, including incidence, risks, causes and management, on HB is scarce in critically ill hematological patients. In order to decipher its burden, we analyzed a large prospective multicenter sample of critically ill hematological patients and report on the management of HB by caring physicians.

\section{Patients And Methods}

\section{Study population}

We performed a post hoc analysis of a Franco-Belgian multicenter prospective study assessing the prognosis of patients with hematological malignancies admitted in 17 ICU between January 2010 and May 2011 (26). Among the 1011 patients enrolled in the original study, only patients with total serum bilirubin measurement at admission were included. Two groups were created according to liver SOFA score (21) at ICU admission: one with HB defined as a liver SOFA score $>1$ (total bilirubinemia $\geq 33$ $\mu \mathrm{mol} / \mathrm{L}$ ) and one without HB defined as a liver SOFA score $\leq 1$ (total bilirubinemia $<33 \mu \mathrm{mol} / \mathrm{L}$ ) (24). The appropriate ethics committees approved the study (26) and all patients or relatives were informed and consented to participate in the study.

\section{Data collected in the prospective cohort}

All patients had a diagnosis of initial/relapsed hematological malignancy within 5 years before ICU admission. The performans status (27) and the Charlson comorbidity index (28) were determined at ICU admission. History of mild, moderate or severe liver diseases was defined by a hepatic Charlson comorbidity index $\geq 1$ (severe = cirrhosis and portal hypertension with variceal bleeding history, moderate = cirrhosis and portal hypertension but no variceal bleeding history, mild = chronic hepatitis or cirrhosis without portal hypertension). Underlying malignancies, disease status at ICU admission, radiotherapy and chemotherapy received in the last month and HSCT were recorded. In allo-HSCT patients, the type of donor, type and intensity of conditioning regimen and GvHD prophylaxis and treatment were collected. Chemotherapy, systemic corticosteroids, hematopoietic growth factors and immunosuppressive drugs prescribed by the hematologist in charge of each patient and administered during ICU stay were recorded. Neutropenia was defined as a neutrophil count of less than $0.5 \mathrm{G} / \mathrm{L}$ and presence of haemophagocytosis features on myelogram was informed. Data from clinical examination at ICU admission were reported 
such as digestive, neurological, cutaneous, renal and hematological symptoms. Organ dysfunctions, presence of sepsis and life sustaining therapies at ICU admission and during the ICU stay were also recorded.

\section{Data collection}

Medical hospital records of patients with HB at ICU admission were selected and reviewed by an expert panel including an intensivist (MB), a hematologist (CS) and hepatologists (CM and VM). The five following questions were assessed: (1) was HB at ICU admission mentioned by the attending physician in the medical records? (2) was a diagnostic strategy carried out to explore HB, including a liver biopsy? (3) which cause of HB was suspected by the attending physician or confirmed by hepatic histology when liver biopsy was performed just before ICU admission or during ICU stay? (4) which specific treatments were initiated? (5) which cause of HB was considered a posteriori by the expert panel? Etiologic diagnoses of HB were established by the expert panel according to pre-established definitions of GvHD (15), SOS (17), haemophagocytic syndrome (19), hypoxic hepatitis (29), sepsis (11),viral hepatitis (30), liver fungal infections (10), drug induced liver injury (31).

\section{Statistical analysis}

Results were reported as median and interquartile ranges or counts and proportions (\%). Qualitative variables were compared using the chi-square test or Fisher's exact test as appropriate and continuous variables using the Mann-Whitney test. Multivariate analyses were performed using logistic regressions. Variables yielding P-values $<0.20$ in the univariate analyses or considered clinically relevant were entered in backward stepwise logistic regression models. Survival curves were constructed according to the Kaplan-Meier method and compared with the log-rank test. P-values $<0.05$ were considered statistically significant. Statistical analyses were done using SPSS software (version 20).

\section{Results}

\section{Characteristics of patients}

Among the 1011 patients included in the TRIALOH study (26), 118 (11.7\%) were excluded because total serum bilirubin level was not measured at ICU admission (Figure 1). Among the 893 remaining patients, 185 (20.7\%) patients had HB, defined as a total bilirubinemia $\geq 33 \mu \mathrm{mol} / \mathrm{L}$ at admission, which corresponds to a SOFA score $>1$.

Characteristics of the cohort by HB are presented in Table 1. Median (interquartile range) age was 60 (49 - 70) years and there was a majority (61\%) of men. Less than $5 \%$ of patients had a history of liver diseases. Non-Hodgkin lymphoma was the most frequent underlying hematological malignancy (31.5\%), followed by acute myeloid leukemia (27.1\%) and myeloma (11.9\%). There were 133 (14.7\%) allo-HSCT patients, including $6.8 \%$ with a previous myeloablative conditioning regimen. About two-third of patients (64.9\%) had been treated with antibiotics before ICU transfer. Acute respiratory failure concerned almost 
two-third (62.4\%) of patients at ICU admission, followed by cardiovascular failure (43.1\%), acute kidney injury (31\%) and acute liver failure (8.8\%). A total of $497(55.7 \%)$ patients had at least two organ failures at ICU admission. Digestive, hematological (such as lymphadenopathy, mucositis, hepatomegaly, splenomegaly, purpura, and intraoral hemorrhage) and/or neurological symptoms were found on clinical examination in one-quarter of patients. Renal symptoms (such as decreased urine output, anuria, hematuria, and pyuria) were present in one-third of patients (32.2\%). Nearly one-third of patients (30.8\%) were neutropenic, sepsis was diagnosed for 578 (64.7\%) patients, and a high majority of patient received antimicrobial therapy at ICU admission and during ICU stay. About $10 \%$ of patients were receiving chemotherapy at ICU admission. Few patients had positive Cytomegalovirus (CMV) or Herpes simplex virus (HSV) nucleic acid testing (NAT). Haemophagocytosis features were described on myelograms in 16 patients $(1.8 \%)$. Half of patients required invasive mechanical ventilation and/or vasoactive drugs during ICU stay, and one-third needed renal replacement therapy.

Table 1. Characteristics of patients 


\begin{tabular}{|c|c|c|c|c|}
\hline & $\begin{array}{l}\text { All } \\
(n=893)\end{array}$ & $\begin{array}{l}\text { With HB } \\
(\mathrm{n}=185)\end{array}$ & $\begin{array}{l}\text { Without HB } \\
(\mathrm{n}=708)\end{array}$ & $p$ \\
\hline Age (years), median [IQR] & $\begin{array}{l}60[49- \\
70]\end{array}$ & $56[47-54]$ & $61[49-70]$ & $<0.0001$ \\
\hline Male sex, n (\%) & $\begin{array}{l}545 \\
(61.0)\end{array}$ & $121(65.4)$ & $424(59.9)$ & 0.171 \\
\hline Performans status >1, n (\%) & $\begin{array}{l}470 \\
(52.6)\end{array}$ & $93(50.3)$ & $377(53.2)$ & 0.47 \\
\hline Charlson comorbidity index, median [IQR] & $4[3-6]$ & $4[2-5]$ & $4[3-6]$ & 0.005 \\
\hline $\begin{array}{l}\text { Hepatic Charlson comorbidity index } \geq 1, n \\
(\%)\end{array}$ & $41(4.6)$ & $15(8.1)$ & $26(3.7)$ & 0.01 \\
\hline SOFA score at admission, median [IQR] & 6 [3-9] & 8 [6-13] & $5[3-8]$ & $<0.0001$ \\
\hline $\begin{array}{l}\text { SOFA score without bilirubin level at } \\
\text { admission, median [IQR] }\end{array}$ & $5[3-8]$ & $6[4-10]$ & $5[3-8]$ & $<0.0001$ \\
\hline \multicolumn{5}{|l|}{ Underlying malignancy, $\mathrm{n}(\%)$} \\
\hline Acute myeloid leukemia & $\begin{array}{l}242 \\
(27.1)\end{array}$ & $56(30.3)$ & $186(26.3)$ & 0.276 \\
\hline Acute lymphocytis leukemia & $64(7.2)$ & $13(7.0)$ & $51(7.2)$ & 0.934 \\
\hline Non-hodgkin lymphoma & $\begin{array}{l}281 \\
(31.5)\end{array}$ & $60(32.4)$ & $221(31.2)$ & 0.751 \\
\hline Hodgkin lymphoma & $23(2.6)$ & $5(2.7)$ & $18(2.5)$ & 0.902 \\
\hline Myeloma & $\begin{array}{l}106 \\
(11.9)\end{array}$ & $11(5.9)$ & $95(13.4)$ & 0.005 \\
\hline Chronic lymphocytic leukemia & $70(7.8)$ & $8(4.3)$ & $65(8.8)$ & 0.046 \\
\hline Chronic myeloid leukemia & $16(1.8)$ & $1(0.5)$ & $15(2.1)$ & 0.15 \\
\hline Myelodysplastic syndrome & $32(3.6)$ & $10(5.4)$ & $22(3.1)$ & 0.134 \\
\hline Other & $59(6.6)$ & $21(11.4)$ & $38(5.4)$ & 0.004 \\
\hline Disease status at ICU admission, $\mathrm{n}(\%)$ & & & & 0.69 \\
\hline No remission/progression & $\begin{array}{l}340 \\
(38.1)\end{array}$ & $63(34.1)$ & $277(39.1)$ & \\
\hline Complete remission & $\begin{array}{l}148 \\
(16.6)\end{array}$ & $36(19.5)$ & $112(15.8)$ & \\
\hline Partial remission & $62(6.9)$ & $13(7.0)$ & $49(6.9)$ & \\
\hline Newly diagnosed malignancy & $\begin{array}{l}214 \\
(24.0)\end{array}$ & $46(24.9)$ & $168(23.7)$ & \\
\hline
\end{tabular}


Unknown

129

(14.4)

27 (14.6)

$102(14.4)$

Chemotherapy in the last 1 month, $\mathrm{n}(\%)$

\begin{tabular}{|c|c|c|c|c|}
\hline Antracyclin & $\begin{array}{l}379 \\
(42.4)\end{array}$ & $91(49.2)$ & $288(40.7)$ & 0.037 \\
\hline Aracytin & $\begin{array}{l}266 \\
(29.8)\end{array}$ & $74(40.0)$ & $192(27.1)$ & 0.001 \\
\hline Cyclophosphamide & $\begin{array}{l}308 \\
(34.5)\end{array}$ & $62(33.5)$ & $246(34.7)$ & 0.945 \\
\hline Bortezomid & $63(7.1)$ & $7(8.8)$ & $56(7.9)$ & 0.051 \\
\hline Busulfan & $17(1.9)$ & $7(3.8)$ & $10(1.4)$ & 0.036 \\
\hline Thalidomide & $44(4.9)$ & $4(2.2)$ & $40(5.6)$ & 0.051 \\
\hline Fludarabin & $88(9.9)$ & $26(14.1)$ & $62(8.8)$ & 0.031 \\
\hline Radiotherapy, n (\%) & $62(7.0)$ & $19(10.3)$ & $43(6.1)$ & 0.048 \\
\hline Allo-HSCT, n (\%) & $\begin{array}{l}131 \\
(14.7)\end{array}$ & $45(24.3)$ & $86(12.2)$ & $<0.0001$ \\
\hline Auto-HSCT, n (\%) & $\begin{array}{l}133 \\
(14.9)\end{array}$ & $28(15.1)$ & $105(14.9)$ & 0.935 \\
\hline $\begin{array}{l}\text { Myeloablative conditioning regimen for allo- } \\
\text { HSCT }\end{array}$ & $61(6.8)$ & $18(9.7)$ & $43(6.1)$ & 0.079 \\
\hline \multicolumn{5}{|l|}{ Treatments before ICU admission, n (\%) } \\
\hline $\begin{array}{l}\text { Systemic corticosteroids the month } \\
\text { before }\end{array}$ & $\begin{array}{l}339 \\
(38.1)\end{array}$ & $76(41.1)$ & $263(37.4)$ & 0.354 \\
\hline Cyclosporin the month before & $73(8.2)$ & $32(17.4)$ & $41(5.8)$ & $<.0001$ \\
\hline $\begin{array}{l}\text { Mycophenolate Mofetil the month } \\
\text { before }\end{array}$ & $45(5.0)$ & $17(8.2)$ & $28(4.0)$ & 0.004 \\
\hline $\begin{array}{l}\text { Antimicrobial therapy the } 10 \text { days } \\
\text { before }\end{array}$ & $\begin{array}{l}579 \\
(64.9)\end{array}$ & $139(75.1)$ & $440(62.2)$ & 0.001 \\
\hline \multicolumn{5}{|l|}{ Organ failure at ICU admission, n (\%) } \\
\hline Acute respiratory failure & $\begin{array}{l}556 \\
(62.4)\end{array}$ & $117(63.2)$ & $439(62.2)$ & 0.791 \\
\hline Cardiovascular failure & $\begin{array}{l}384 \\
(43.1)\end{array}$ & $84(45.4)$ & $300(42.5)$ & 0.473 \\
\hline Acute kidney injury & $\begin{array}{l}276 \\
(31.0)\end{array}$ & $67(36.2)$ & $209(29.6)$ & 0.083 \\
\hline Acute hepatic failure & $78(8.8)$ & $43(23.2)$ & $35(5.0)$ & $<.0001$ \\
\hline
\end{tabular}


Coagulopathy

Neurological failure

Multi-Organ failure
181

(20.3)

202

(22.6)

497

(55.7)
$52(28.1)$

47 (25.4)

$123(66.5)$

$375(52.9)$

0.001

Clinical situations at ICU admission, n (\%)

Digestive symptoms

$\begin{array}{llll}215 & 75(40.5) & 140(20.0) & <0.0001\end{array}$

Jaundice

$34(3.8)$

32 (17.3)

$2(0.3)$

$<0.0001$

Diarrhea

$91(10.2) \quad 23(12.4)$

68 (9.6)

0.258

Gastrointestinal bleeding

Abdominal pain

$16(1.8) \quad 6(3.2)$

$10(1.4)$

0.095

123

(13.8)

Occlusive syndrome

$28(3.1)$

$39(21.1)$

$84(11.9)$

0.001

Ascites

$29(3.2)$

Renal symptoms

285

(32.2)

Tumor lysis syndrome

87 (9.7)

7 (3.8)

$21(3.0)$

0.57

Hematological symptoms

Hepatomegaly

Splenomegaly

Mucositis

Neurological symptoms

Neutropenia

Sepsis

233

(26.3)

89 (10.0) $\quad 27(14.6)$

$62(8.8)$

0.018

$78(8.7)$

$22(11.9)$

$56(7.9)$

0.088

$36(4.0)$

$13(7.0)$

$23(3.2)$

0.02

221

(24.9)

262

(30.8)

578

(64.7)

Treatment received at ICU admission, $\mathrm{n}(\%)$

Antimicrobial therapy

732

(82.0)

Cyclosporin

Chemotherapy

$51(27.6)$

$76(42.7)$

$119(64.3)$

$170(24.2)$

0.349

$186(27.6)$

$<0.0001$

0.898

$459(64.8)$ 


\begin{tabular}{|c|c|c|c|c|}
\hline Systemic corticosteroids & $\begin{array}{l}173 \\
(19.4)\end{array}$ & $36(19.5)$ & $137(19.4)$ & 0.98 \\
\hline Stress dose corticosteroids & $\begin{array}{l}159 \\
(17.8)\end{array}$ & $36(19.5)$ & $132(17.4)$ & 0.514 \\
\hline \multicolumn{5}{|l|}{$\begin{array}{l}\text { Life sustaining therapies at ICU admission, } \\
\mathrm{n}(\%)\end{array}$} \\
\hline Invasive mechanical ventilation & $\begin{array}{l}261 \\
(29.2)\end{array}$ & $60(32.4)$ & $201(28.4)$ & 0.282 \\
\hline Noninvasive mechanical ventilation & $\begin{array}{l}149 \\
(16.4)\end{array}$ & $40(21.6)$ & $109(15.4)$ & 0.043 \\
\hline Vasoactive drugs & $\begin{array}{l}297 \\
(33.3)\end{array}$ & $72(98.9)$ & $225(31.8)$ & 0.068 \\
\hline Renal replacement therapy & $\begin{array}{l}106 \\
(11.9)\end{array}$ & $29(15.7)$ & $77(10.9)$ & 0.072 \\
\hline \multicolumn{5}{|l|}{ Laboratory testing at ICU admission } \\
\hline Total Bilirubinemia, $\mu \mathrm{mol} / \mathrm{L}$ & $14[8-26]$ & $57[42-100]$ & 12 [8-18] & $<0.0001$ \\
\hline Aspartate aminotransferase, $x$ ULN & $1[1-2]$ & $2[1-3.9]$ & $1[1-1]$ & $<0.0001$ \\
\hline Alanine aminotransferase, $x$ ULN & $1[1-1.7]$ & $1.4[1-2.9]$ & $1[1-1.4]$ & $<0.0001$ \\
\hline Gamma glutamyltranspeptidase, x ULN & $3.5]$ & $2.4[1-5.4]$ & $1.4[1-3]$ & $<0.0001$ \\
\hline Alkaline phosphatase, $x$ ULN & $1[1-1]$ & $1[1-2.3]$ & $1[1-1]$ & $<0.0001$ \\
\hline Prothrombin Time, \% & $\begin{array}{l}64 \text { [51- } \\
77]\end{array}$ & 57 [44-73] & $66[53-79]$ & $<0.0001$ \\
\hline Platelets, G/L & $\begin{array}{l}62 \text { [30- } \\
147]\end{array}$ & 39 [18-72] & 71 [32-157] & $<0.0001$ \\
\hline Serum creatinine, $\mu \mathrm{mol} / \mathrm{L}$ & $\begin{array}{r}102[69- \\
170]\end{array}$ & $\begin{array}{l}126[80- \\
206]\end{array}$ & 98 [67-156] & $<0.0001$ \\
\hline Hemoglobin, g/dL & $\begin{array}{l}9.1[8- \\
10.6]\end{array}$ & $\begin{array}{l}8.7[7.9- \\
10.2]\end{array}$ & $9.2[8-10.6]$ & 0.062 \\
\hline Leucocytes, G/L & $\begin{array}{l}5.5[0.8- \\
15.3]\end{array}$ & $\begin{array}{l}2.3[0.3- \\
12.3]\end{array}$ & $6.0[11-17]$ & $<0.0001$ \\
\hline Bicarbonate concentration, $\mathrm{mmol} / \mathrm{L}$ & $\begin{array}{l}22[18- \\
25]\end{array}$ & 21 [17-25] & 22 [18-25] & 0.038 \\
\hline Lactate, $\mathrm{mmol} / \mathrm{L}$ & ${ }_{4.2]}^{2.1}{ }^{[1.2-}$ & $2.9[1.6-5.9]$ & $2.0[1.2-4.1]$ & 0.001 \\
\hline Positive CMV NAT & $46(5.2)$ & $19(10.3)$ & $27(3.8)$ & $<0.0001$ \\
\hline Positive HSV NAT & $30(3.4)$ & $13(7.0)$ & $17(2.4)$ & 0.002 \\
\hline
\end{tabular}


Life-Sustaining therapies during ICU stay, n

(\%)

Noninvasive mechanical ventilation

265

(29.7)

$62(33.5) \quad 203(28.7)$

0.199

Invasive mechanical ventilation

441

(49.4)

$110(59.5) \quad 331(46.8) \quad 0.002$

Vasoactive drugs

470

(52.6)

Renal replacement therapy

253

(28.4)

$117(63.2)$

353 (49.9)

0.001

Treatment received during ICU stay, n (\%)

Antibiotic treatment

815

(91.3)

Antifungal treatment

357

(40.0)

Antiviral treatment

385

(43.1)

Stress dose corticosteroids

550

(61.6)

Hematopoietic growth factors

\begin{tabular}{|lllll|}
\hline Antibiotic treatment & $\begin{array}{l}815 \\
(91.3)\end{array}$ & $173(93.5)$ & $642(90.7)$ & 0.224 \\
\hline Antifungal treatment & $\begin{array}{l}357 \\
(40.0)\end{array}$ & $92(49.7)$ & $265(37.4)$ & 0.002 \\
Antiviral treatment & $\begin{array}{l}385 \\
(43.1)\end{array}$ & $88(47.6)$ & $297(41.9)$ & 0.169 \\
Stress dose corticosteroids & $\begin{array}{l}550 \\
(61.6)\end{array}$ & $119(64.3)$ & $431(60.9)$ & 0.391 \\
\hline Hematopoietic growth factors & $\begin{array}{l}155 \\
(17.4)\end{array}$ & $43(23.2)$ & $112(15.8)$ & 0.018 \\
\hline Nosocomial infection during ICU stay, $\mathrm{n}(\%)$ & $\begin{array}{l}152 \\
(17.0)\end{array}$ & $48(25.9)$ & $104(14.7)$ & $<0.0001$ \\
\hline
\end{tabular}

(17.4)

Nosocomial infection during ICU stay, n (\%)

\begin{tabular}{|lllll|}
\hline Antibiotic treatment & $\begin{array}{l}815 \\
(91.3)\end{array}$ & $173(93.5)$ & $642(90.7)$ & 0.224 \\
\hline Antifungal treatment & $\begin{array}{l}357 \\
(40.0)\end{array}$ & $92(49.7)$ & $265(37.4)$ & 0.002 \\
Antiviral treatment & $\begin{array}{l}385 \\
(43.1)\end{array}$ & $88(47.6)$ & $297(41.9)$ & 0.169 \\
Stress dose corticosteroids & $\begin{array}{l}550 \\
(61.6)\end{array}$ & $119(64.3)$ & $431(60.9)$ & 0.391 \\
\hline Hematopoietic growth factors & $\begin{array}{l}155 \\
(17.4)\end{array}$ & $43(23.2)$ & $112(15.8)$ & 0.018 \\
\hline Nosocomial infection during ICU stay, $\mathrm{n}(\%)$ & $\begin{array}{l}152 \\
(17.0)\end{array}$ & $48(25.9)$ & $104(14.7)$ & $<0.0001$ \\
\hline
\end{tabular}

$174(24.6)$

$<0.0001$

$79(42.7) \quad 174(24.6) \quad<0.0001$

Note: HB was defined as total serum bilirubin level $\geq 33 \mu \mathrm{mol} / \mathrm{L}$. (ICU: Intensive care unit; HB:

hyperbilirubinemia; SOFA score: Sepsis-related Organ Failure Assessment score; Allo-HSCT: allogenic hematopoietic stem cell transplantation; Auto-HSCT: autologous hematopoietic stem cell transplantation; CMV: Cytomegalovirus; HSV: Herpes simplex virus; NAT: Nucleic acid testing; UNL: upper limit of normality)

\section{HB and outcome}

ICU mortality and hospital mortality were $(n=84) 45.4 \%$ and $(n=103) 56.3 \%$ and $(n=175) 24.7 \%$ and $(n=254) 36.3 \%$ for patients with and without HB. HB was associated with hospital mortality (odd ratio $[\mathrm{OR}]=2.26,95 \% \mathrm{Cl}=1.62-3.14, \mathrm{p}<0.0001$ and Figure 2). After adjustment for invasive mechanical ventilation (MV), renal replacement therapy (RRT) and vasoactive drugs, HB remained an independent factor associated with hospital mortality (adjusted OR=1.86, 95\% Cl=1.28 - 2.72, p=0.001). Median ICU and hospital lengths of stay were not different for patients with and without HB. Independent risk factors for hospital mortality among HB patients were hepatic Charlson comorbidity index $\geq 1(\mathrm{OR}=11.67,95 \%$ $\mathrm{Cl}=1.37-99.72, \mathrm{p}=0.025)$, performance status $>1(\mathrm{OR}=2.14,95 \% \mathrm{Cl}=1.04-4.41, \mathrm{p}=0.040)$, acute respiratory 
failure (ARF) at before ICU admission (OR=2.23, 95\% $\mathrm{Cl}=1.03-4.82, \mathrm{p}=0.042)$, mechanical ventilation (MV) during ICU stay $(O R=4.84,95 \% \mathrm{Cl}=2.24-10.46, p<0.0001)$, RRT during ICU stay $(\mathrm{OR}=2.34,95 \% \mathrm{Cl}=1.08$ 5.07, $p=0.031)$, and chemotherapy initiated at ICU admission ( $O R=0.23,95 \% \mathrm{Cl}=0.06-0.79, p=0.020)$. The univariate analyses are presented in additional table 1.

\section{Risk factors for HB in critically ill hematological patients at ICU admission}

Patients with HB were younger than patients without HB; had more frequently hepatic comorbidities; had received more anthracyclins, aracytin, busulfan but not cyclophosphamide, thalidomide and fludarabine; had undergone more frequently allo-HSCT; were treated with cyclosporine, mycophenolate mofetil or antimicrobial therapy; presented with more organ failures and digestive clinical symptoms; had higher serum concentrations of hepatic enzymes, a longer prothrombin time, lower platelet and higher lactate levels; and required more life sustaining therapies during ICU stay. Factors independently associated with HB at ICU admission are presented in Table 2. The presence at ICU admission of cyclosporin treatment, antimicrobial therapy, digestive symptoms, ascites, history of liver diseases, neutropenia and increased serum creatinine level, were associated with an increased risk of HB. Myeloma and its treatments were not associated with a higher risk of HB.

Table 2. Factors independently associated with HB in critically ill hematological patients

\begin{tabular}{|llll|}
\hline & OR & $95 \% \mathrm{Cl}$ & $p$ \\
\hline Cyclosporin the month before ICU admission & 3.357 & $1.926-5.851$ & $<0.0001$ \\
\hline Antimicrobial treatment before ICU admission & 1.578 & $1.038-2.401$ & 0.033 \\
\hline Digestive symptoms at ICU admission & 2.182 & $1.461-3.258$ & $<0.0001$ \\
\hline Hepatic Charlson comorbidity index $\geq 1$ & 2.228 & $1.057-4.696$ & 0.035 \\
\hline Ascites at ICU admission & 2.562 & $1.059-6.196$ & 0.037 \\
\hline Increased serum creatinine level at ICU admission & 1.001 & $1.000-1.003$ & 0.021 \\
\hline Neutropenia at ICU admission & 1.465 & $1.001-2.144$ & 0.049 \\
\hline Myeloma & 0.378 & $0.188-0.761$ & 0.006 \\
\hline
\end{tabular}

Note: (HB: hyperbilirubinemia; ICU: Intensive Care Unit; Cl: confidence interval). Hepatic Charlson comorbidity index was defined as cirrhosis +/- portal hypertension +/- variceal bleeding history.

\section{Management of hematological patients with HB in the ICU}

A total of $173(93.5 \%)$ medical records of HB patients were reviewed. HB was overlooked by the caring physician in 92 (53\%) patients. Otherwise, a liver workup was performed in 51 (63\%) patients among 81 
(47\%) for whom HB at admission was reported in the medical record. The diagnostic strategies included one or more of the following tests: liver ultrasonography, abdominal CT scan, serology and hepatotropic viruses and/or herpes viruses NAT, liver biopsies, echocardiography, bone marrow aspirate or bone marrow biopsy, biological markers of hemolysis and haemophagocytic syndrome. Causes of HB were evoked by the caring physician for $48(59 \%)$ patients (Table 3 ). Among these patients, $32(40 \%)$ received a specific treatment for HB such as chemotherapy for $16(19.8 \%)$ patients, discontinuation of cyclosporin for $4(4.9 \%)$ patients, systemic corticosteroids for three (3.7\%) patients with hemolysis and one $(1.2 \%)$ patient with GvHD, introduction of dobutamine for three (3.7\%) patients, antimicrobial treatment for three (3.7\%) patients, anti-fibrinolytic for one (1.2\%) patient with SOS, hepatic arterial embolization for one $(1.2 \%)$ patient with active hepatic bleeding. Among patients with HB, liver biopsy was performed in nine (5.2\%) patients just before ICU admission or during ICU stay. A liver biopsy was performed transcutaneously in five $(2.9 \%)$ cases, post-mortem in two $(1.2 \%)$ cases, intraoperatively in $1(0.6 \%)$ case and with an unknown method in one $(0.6 \%)$ case. Complications due to percutaneous liver biopsies occurred in two patients, including intrahepatic and intra-peritoneal hemorrhages, which led to hemorrhagic shock and death for one of them. No transjugular liver biopsy was performed during the study period. Hepatic histological findings, available for eight biopsies, were one CMV viral hepatitis, one blastic infiltration, one lymphoma infiltration, one neuroendocrine tumor with liver infiltration, one toxic or drug origin necrosis cell with steatosis, one thrombotic microangiopathy with haemophagocytis, one SOS with GvHD injury, and one extramedullary haematopoiesis. The expert panel reclassified a posteriori 65 (37.6\%) patients (Table 3), including 21 (12\%) patients that could have had access to a specific treatment.

Table 3. Causes of HB at ICU admission in a cohort of 893 critically ill hematologic patients 


\begin{tabular}{|c|c|c|c|}
\hline & Caring physician & $n=173$ & Reviewers* $n=173$ \\
\hline Specific infiltration, $\mathrm{n}(\%)$ & $11(6.4)$ & & $18(10.4)$ \\
\hline Drug toxicity, n (\%) & $10(5.8)$ & & $7(4.0)$ \\
\hline Hypoxic hepatitis, n (\%) & $6(3.5)$ & & $12(6.9)$ \\
\hline Haemophagocytis syndrome, n (\%) & $4(2.3)$ & & $9(5.2)$ \\
\hline Infectious hepatitis, n (\%) & $3(1.7)$ & & $8(4.6)$ \\
\hline Haemolysis, n (\%) & $3(1.7)$ & & $3(1.7)$ \\
\hline GvHD, n (\%) & $2(1.2)$ & & $7(4.0)$ \\
\hline Sinusoidal obstruction syndrome, n (\%) & $2(1.2)$ & & $3(1.7)$ \\
\hline Multi-organ failure, $\mathrm{n}(\%)$ & $1(0)$ & & $5(2.9)$ \\
\hline Sepsis, n (\%) & $0(0)$ & & $4(2.3)$ \\
\hline Other causes, n (\%) & $6(3.5) \S$ & & $4(4.0)^{¥}$ \\
\hline Undetermined, n (\%) & $33(19)$ & & $93(53.8)$ \\
\hline No liver workup, $n(\%)$ & 92 (53.2\%) & & - \\
\hline
\end{tabular}

Note: (GvHD: Graft versus host disease). * A panel of experts composed of an intensivist, a hematologist and a hepatologist. § other causes were: $n=1$ thrombotic microangiopathy, $n=1 \mathrm{AL}$ amyloidosis, $n=1$ active right hepatic arterial bleeding, $n=1$ cirrhosis decompensation, $n=1$ neuroendocrine tumor with liver infiltration, $\mathrm{n}=1$ left biliary dilatation without obstructive etiology; $¥$ other causes were: $n=1$ thrombotic microangiopathy (histological finding), $n=1$ active right hepatic arterial bleeding, $n=1$ cirrhosis decompensation, $n=1$ neuroendocrine tumor with liver infiltration.

\section{Discussion}

In a large multicentric cohort, HB, defined as a total serum bilirubin level $\geq 33 \mu \mathrm{mol} / \mathrm{L}$ (twice the upper limit of normal range), concerned $21 \%$ of critically ill patients with hematological malignancy at ICU admission and was associated with outcome. The first underlying causes of HB were cancer progression, hypoxic hepatitis, cancer treatment toxicities, and sepsis. Liver workups for HB, including liver biopsies, led to high rates of treatment modifications, including cancer treatment, which was associated with reduced mortality. In spite of that, HB was overlooked by the intensive care teams in more than half of patients.

This is the largest study reporting on the burden of HB in critically ill hematological patients. Our findings demonstrate that $\mathrm{HB}$ is closely related to the prognosis hematological patients, as for other critical care 
patients, including severely burned patients (32), patients with acute respiratory distress syndrome (ARDS) (33) and trauma (34). An identical serum bilirubin threshold, twice the upper limit of normal range, has been associated with an increased risk of mortality in patients with severe sepsis and septic shock (35).

There is, to date, no robust literature on the underlying causes of HB in critically ill hematological patients. In this series, almost one third of treatable liver diseases contributed to HB. In the rare subgroup of patients who underwent a throughout liver workup, cancer, cancer drug toxicities, heart failures and infections led frequently to therapeutic adjustments, including antimicrobial treatment modifications, chemotherapy initiations, cancer drug modifications, hemodynamic management, and defibrotide in patients with SOS.

We were surprised by the rather high number of patients in whom HB was overlooked in this cohort: total serum bilirubin was not measured in $12 \%$ of patients at ICU admission, was unrecognized in half of HB patients, and was poorly investigated $(63 \%)$, diagnosed $(59 \%)$ and treated $(40 \%)$ when it was taken into account by the intensive care team. HB is also frequently ignored in other immunocompetent critically ill patients, although it is closely associated with outcome $(36,37)$. Our findings support the idea that HB is not a satellite of the multi-organ failure syndrome and that it should be actively treated as other organ failures. Echocardiography, bacterial, virologic and fungal workups, liver ultrasonography, CT-scans, and bone marrow aspirate or biopsy should be performed immediately at bedside of HB patients. We acknowledge the absence of guideline to support empirical treatments or decide when to perform liver biopsies in hematological HB patients. The few numbers of liver biopsies performed in the cohort could have modified treatments. Percutaneous liver biopsy is contraindicated in the context of severe thrombopenia and was associated with mortality in this series. Transjugular liver biopsy is safe $(38,39)$ and more than 20 years ago, Shulman at al. demonstrated that it significantly improved the management of allo-HSHC patients (40).

The presence at ICU admission of cyclosporin treatment, antimicrobial therapy, digestive symptoms, ascites, history of liver diseases, increased serum creatinine level, neutropenia were associated with HB. Cyclosporin treatment was strongly associated with $\mathrm{HB}$, which confirmed the relationship between alloHSCT and outcome of hematological patients (41). Other classical factors were neutropenia, favoring sepsis and requiring antimicrobial therapy. In hematological patients, HB and acute kidney injury (AKI) seem to share similar risk factors such as sepsis, antimicrobials and cyclosporin nephrotoxicity, tumor lysis syndrome (42), except chemotherapy which did not impact HB in our cohort. Ascites can be explained in these patients by SOS (17), engraftment syndrome in allo-HSCT patients (43), capillary leak syndrome (44) which can also be causes of AKI.

Our study is limited by its heterogeneity, including underlying malignancies, cancer treatments, disease status at admission, and by the high proportion of patients that did not undergo a complete liver workup, including liver biopsy. Its retrospective nature limited our conclusions because HB etiologies may vary widely between subgroups of patients. The absence of systematic NAT testing for hepatotropic viruses of 
HB patients, including HEV (45), is an example, and underlines the importance to update management recommendations (30). There are currently no consensual ICU admission criteria for hematological patients. Admission differs according to center's experience, hematologist involvement in the ICU and case-volume effect (46), therefore the timing between HB onset and admission to the ICU varied between patients. Nevertheless, our results suggest that HB patients should be evaluated for ICU admission. Our results could pave the way for recommendations on $\mathrm{HB}$ in hematological patients. Early recognition of HB should be the first step, as for acute respiratory failure, which is easily and early diagnosed when oxygen begins to be necessary. Systematic diagnostic strategies for HB should be developed and validated in hematological patients, as for acute respiratory failure (47). It is well shown that an absence of etiological diagnosis impairs prognosis of patients with acute respiratory failure (48). It seems to be the same for HB.

\section{Conclusions}

$\mathrm{HB}$ is common, underestimated, infrequently investigated, and is associated with outcome of critically ill hematological patients. HB should be considered upon ICU admission and managed as other organ dysfunctions. Collaborative and multidisciplinary clinical and research networks are crucial both to improve our understanding of HB pathogenesis and to develop diagnostic strategies and adapted therapeutic options, as well as prevention of liver injury. It implies an accurate severity assessment at ICU admission and a close collaboration between hematologists, intensivists and hepatologists. In this setting transjugular liver biopsy needs to be evaluated.

\section{Abbreviations}

HB: Hyperbilirubinemia

ICU: Intensive care unit

HSCT: Hematopoietic stem cell transplantation

Allo-HSCT: Allogenic hematopoietic stem cell transplantation

Auto-HSCT: Autologous hematopoietic stem cell transplantation

DILI: Drug-induced liver injury

SOS: Sinusoidal obstruction syndrome

GvHD: Graft-versus-host disease

SOFA score: Sepsis-related Organ Failure Assessment score

CMV: Cytomegalovirus 
HSV: Herpes simplex virus

NAT: Nucleic acid testing

MV: Invasive mechanical ventilation

RRT: Renal replacement therapy

ARF: Acute respiratory failure

AKI: Acute kidney injury

UNL: Upper limit of normal range

ARDS: Acute respiratory distress syndrome

\section{Declarations}

Ethics approval and consent to participate: The study was approved by the appropriate ethics committees in France and Belgium. This statement is in "patients and methods" paragraph.

Consent for publication: Not applicable.

Availability of data and materials: The datasets used and/or analyzed during the current study are available from the corresponding author on reasonable request.

Competing interests: The authors declare that they have no competing interests.

Funding: The authors declare that they have no funding for this research.

Authors' contributions: $M B, M D, D M$ and EA determined the study concept and design. AE, DM, MD, VL, FP, $A K, A D, F V, M N, F B, C L, A R, A P M, D B, R H, M J$ acquired the prospective data. $D M, M D$ and $M B$ interpreted the data and performed the statistical analyses. MB, VM, CS and CM drafted the manuscript. All authors read and approved the final manuscript.

Acknowledgements: Not applicable.

\section{References}

1. McDonald GB. Hepatobiliary Complications of Hematopoietic Cell Transplantation, 40 Years On. Hepatology. 2010 Apr;51(4):1450-60.

2. Tuncer $\mathrm{HH}$. Gastrointestinal and hepatic complications of hematopoietic stem cell transplantation. World J Gastroenterol. 2012;18(16):1851. 
3. on behalf of the Society for Immunotherapy of Cancer Toxicity Management Working Group, Puzanov I, Diab A, Abdallah K, Bingham CO, Brogdon C, et al. Managing toxicities associated with immune checkpoint inhibitors: consensus recommendations from the Society for Immunotherapy of Cancer (SITC) Toxicity Management Working Group. J Immunother Cancer [Internet]. 2017 Dec [cited 2019 Jun 20];5(1). Available from: https://jitc. biomedcentral.com/articles/10.1186/s40425-0170300-z

4. Champiat S, Lambotte O, Barreau E, Belkhir R, Berdelou A, Carbonnel F, et al. Management of immune checkpoint blockade dysimmune toxicities: a collaborative position paper. Ann Oncol. 2016 Apr;27(4):559-74.

5. Lambert J, Pautas C, Terré C, Raffoux E, Turlure P, Caillot D, et al. Gemtuzumab ozogamicin for de novo acute myeloid leukemia: final efficacy and safety updates from the open-label, phase III ALFA0701 trial. Haematologica. 2019 Jan;104(1):113-9.

6. McDonald GB, Slattery JT, Bouvier ME, Ren S, Batchelder AL, Kalhorn TF, et al. Cyclophosphamide metabolism, liver toxicity, and mortality following hematopoietic stem cell transplantation. Blood. 2003 Mar 1;101(5):2043-8.

7. Patel B, Kirkwood AA, Dey A, Marks DI, McMillan AK, Menne TF, et al. Pegylated-asparaginase during induction therapy for adult acute lymphoblastic leukaemia: toxicity data from the UKALL14 trial. Leukemia. 2017;31(1):58-64.

8. Bath RK, Brar NK, Forouhar FA, Wu GY. A review of methotrexate-associated hepatotoxicity: Methotrexate hepatotoxicity. J Dig Dis. 2014 Oct;15(10):517-24.

9. Majhail NS, Lazarus HM, Burns LJ. Iron overload in hematopoietic cell transplantation. Bone Marrow Transplant. 2008 Jun;41(12):997-1003.

10. Fiore M, Cascella M, Bimonte S, Maraolo AE, Gentile I, Schiavone V, et al. Liver fungal infections: an overview of the etiology and epidemiology in patients affected or not affected by oncohematologic malignancies. Infect Drug Resist. 2018;11:177-86.

11. Jenniskens $M$, Langouche L, Vanwijngaerden Y-M, Mesotten D, Van den Berghe G. Cholestatic liver (dys)function during sepsis and other critical illnesses. Intensive Care Med. 2016 Jan;42(1):16-27.

12. Naini BV, Lassman CR. Total parenteral nutrition therapy and liver injury: a histopathologic study with clinical correlation. Hum Pathol. 2012 Jun;43(6):826-33.

13. Povsic M, Wong OY, Perry R, Bottomley J. A Structured Literature Review of the Epidemiology and Disease Burden of Non-Alcoholic Steatohepatitis (NASH). Adv Ther [Internet]. 2019 May 7 [cited 2019 Jun 20]; Available from: http://link.springer.com/10.1007/s12325-019-00960-3

14. Walz-Mattmüller R, Horny H-P, Ruck P, Kaiserling E. Incidence and Pattern of Liver Involvement in Haematological Malignancies. Pathol - Res Pract. 1998 Jan;194(11):781-9.

15. on behalf of the EBMT (European Society for Blood and Marrow Transplantation) Transplant Complications Working Party and the "EBMT-NIH (National Institutes of Health)-CIBMTR (Center for International Blood and Marrow Transplant Research) GvHD Task Force", Schoemans HM, Lee SJ, Ferrara JL, Wolff D, Levine JE, et al. EBMT-NIH-CIBMTR Task Force position statement on 
standardized terminology \& guidance for graft-versus-host disease assessment. Bone Marrow Transplant. 2018 Nov;53(11):1401-15.

16. Corbacioglu S, Jabbour EJ, Mohty M. Risk Factors for Development of and Progression of Hepatic Veno-Occlusive Disease/Sinusoidal Obstruction Syndrome. Biol Blood Marrow Transplant [Internet]. 2019 Feb [cited 2019 Jun 20]; Available from: https://linkinghub.elsevier.com/retrieve/pii/S1083879119301430

17. Roeker LE, Kim HT, Glotzbecker B, Nageshwar P, Nikiforow S, Koreth J, et al. Early Clinical Predictors of Hepatic Veno-Occlusive Disease/Sinusoidal Obstruction Syndrome after Myeloablative Stem Cell Transplantation. Biol Blood Marrow Transplant. 2019 Jan;25(1):137-44.

18. Belay Y, Yirdaw K, Enawgaw B. Tumor Lysis Syndrome in Patients with Hematological Malignancies. J Oncol. 2017;2017:1-9.

19. La Rosée P, Horne A, Hines M, von Bahr Greenwood T, Machowicz R, Berliner N, et al. Recommendations for the management of hemophagocytic lymphohistiocytosis in adults. Blood. 2019 Jun 6;133(23):2465-77.

20. Sorror ML, Maris MB, Storb R, Baron F, Sandmaier BM, Maloney DG, et al. Hematopoietic cell transplantation (HCT)-specific comorbidity index: a new tool for risk assessment before allogeneic HCT. Blood. 2005 Oct 15;106(8):2912-9.

21. Vincent JL, Moreno R, Takala J, Willatts S, De Mendonça A, Bruining H, et al. The SOFA (Sepsisrelated Organ Failure Assessment) score to describe organ dysfunction/failure. On behalf of the Working Group on Sepsis-Related Problems of the European Society of Intensive Care Medicine. Intensive Care Med. 1996 Jul;22(7):707-10.

22. Gooley TA, Rajvanshi P, Schoch HG, McDonald GB. Serum bilirubin levels and mortality after myeloablative allogeneic hematopoietic cell transplantation. Hepatol Baltim Md. 2005 Feb;41(2):345-52.

23. Barba P, Martino R, Perez-Simón JA, Fernández-Avilés F, Piñana JL, Valcárcel $D$, et al. Incidence, characteristics and risk factors of marked hyperbilirubinemia after allogeneic hematopoietic cell transplantation with reduced-intensity conditioning. Bone Marrow Transplant. 2012 Oct;47(10):1343-9.

24. Radhakrishnan K, Bishop J, Jin Z, Kothari K, Bhatia M, George D, et al. Risk factors associated with liver injury and impact of liver injury on transplantation-related mortality in pediatric recipients of allogeneic hematopoietic stem cell transplantation. Biol Blood Marrow Transplant J Am Soc Blood Marrow Transplant. 2013 Jun;19(6):912-7.

25. Thorvaldson L, Remberger M, Winiarski J, Omazic B, Fischler B, Sundin M. HLA, GVHD, and parenteral nutrition are risk factors for hepatic complications in pediatric HSCT. Pediatr Transplant. 2016 Feb;20(1):96-104.

26. Azoulay E, Mokart D, Pène F, Lambert J, Kouatchet A, Mayaux J, et al. Outcomes of Critically III Patients With Hematologic Malignancies: Prospective Multicenter Data From France and Belgium-A 
Groupe de Recherche Respiratoire en Réanimation Onco-Hématologique Study. J Clin Oncol. 2013 Aug;31(22):2810-8.

27. Soares M, Salluh JIF, Carvalho MS, Darmon M, Rocco JR, Spector N. Prognosis of Critically III Patients With Cancer and Acute Renal Dysfunction. J Clin Oncol. 2006 Aug 20;24(24):4003-10.

28. Charlson ME, Pompei P, Ales KL, MacKenzie CR. A new method of classifying prognostic comorbidity in longitudinal studies: development and validation. J Chronic Dis. 1987;40(5):373-83.

29. Van den broecke A, Van Coile L, Decruyenaere A, Colpaert K, Benoit D, Van Vlierberghe H, et al. Epidemiology, causes, evolution and outcome in a single-center cohort of 1116 critically ill patients with hypoxic hepatitis. Ann Intensive Care [Internet]. 2018 Dec [cited 2019 Sep 10];8(1). Available from: https://annalsofintensivecare.springeropen.com/articles/10.1186/s13613-018-0356-z

30. Mallet V, van Bömmel F, Doerig C, Pischke S, Hermine O, Locasciulli A, et al. Management of viral hepatitis in patients with haematological malignancy and in patients undergoing haemopoietic stem cell transplantation: recommendations of the 5th European Conference on Infections in Leukaemia (ECIL-5). Lancet Infect Dis. 2016 May;16(5):606-17.

31. Drug-induced Liver Injury (DILI) Study Group, Chinese Society of Hepatology (CSH), Chinese Medical Association (CMA), Yu Y, Mao Y, Chen C, et al. CSH guidelines for the diagnosis and treatment of drug-induced liver injury. Hepatol Int. 2017 May;11(3):221-41.

32. de Tymowski C, Dépret $F$, Soussi $S$, Nabila M, Vauchel T, Chaussard M, et al. Contributing factors and outcomes of burn-associated cholestasis. J Hepatol. 2019 Sep;71(3):563-72.

33. Dizier S, Forel J-M, Ayzac L, Richard J-C, Hraiech S, Lehingue S, et al. Early Hepatic Dysfunction Is Associated with a Worse Outcome in Patients Presenting with Acute Respiratory Distress Syndrome: A Post-Hoc Analysis of the ACURASYS and PROSEVA Studies. Groeneveld ABJ, editor. PLOS ONE. 2015 Dec 4;10(12):e0144278.

34. Harbrecht BG, Zenati MS, Doyle HR, McMichael J, Townsend RN, Clancy KD, et al. Hepatic Dysfunction Increases Length of Stay and Risk of Death after Injury: J Trauma Inj Infect Crit Care. 2002 Sep;53(3):517-23.

35. Patel JJ, Taneja A, Niccum D, Kumar G, Jacobs E, Nanchal R. The Association of Serum Bilirubin Levels on the Outcomes of Severe Sepsis. J Intensive Care Med. 2015 Jan;30(1):23-9.

36. Lescot T, Karvellas C, Beaussier M, Magder S. Acquired Liver Injury in the Intensive Care Unit: Anesthesiology. 2012 Oct;117(4):898-904.

37. Jenniskens M, Langouche L, Van den Berghe G. Cholestatic Alterations in the Critically III. Chest. 2018 Mar;153(3):733-43.

38. Kis B, Pamarthi V, Fan C-M, Rabkin D, Baum RA. Safety and Utility of Transjugular Liver Biopsy in Hematopoietic Stem Cell Transplant Recipients. J Vasc Interv Radiol. 2013 Jan;24(1):85-9.

39. Ruggiu M, Bedossa P, Rautou PE, Bertheau P, Plessier A, Peffault de Latour R, et al. Utility and Safety of Liver Biopsy in Patients with Undetermined Liver Blood Test Anomalies after Allogeneic Hematopoietic Stem Cell Transplantation: A Monocentric Retrospective Cohort Study. Biol Blood Marrow Transplant. 2018 Dec;24(12):2523-31. 
40. Shulman HM, Gooley T, Dudley MD, Kofler T, Feldman R, Dwyer D, et al. Utility of transvenous liver biopsies and wedged hepatic venous pressure measurements in sixty marrow transplant recipients. Transplantation. 1995 Apr 15;59(7):1015-22.

41. on behalf the Groupe de Recherche en Réanimation Respiratoire du patient d'Onco-Hématologie (GRRR-OH), Saillard C, Darmon M, Bisbal M, Sannini A, Chow-Chine L, et al. Critically ill allogenic HSCT patients in the intensive care unit: a systematic review and meta-analysis of prognostic factors of mortality. Bone Marrow Transplant. 2018 Oct;53(10):1233-41.

42. Rosner MH, Perazella MA. Acute Kidney Injury in Patients with Cancer. Ingelfinger JR, editor. N Engl J Med. 2017 May 4;376(18):1770-81.

43. Spitzer TR. Engraftment syndrome: double-edged sword of hematopoietic cell transplants. Bone Marrow Transplant. 2015 Apr;50(4):469-75.

44. Siddall E, Khatri M, Radhakrishnan J. Capillary leak syndrome: etiologies, pathophysiology, and management. Kidney Int. 2017 Jul;92(1):37-46.

45. von Felden J, Alric L, Pischke S, Aitken C, Schlabe S, Spengler U, et al. The burden of hepatitis E among patients with haematological malignancies: A retrospective European cohort study. $J$ Hepatol. 2019 Sep;71(3):465-72.

46. Zuber B, Tran T-C, Aegerter P, Grimaldi D, Charpentier J, Guidet B, et al. Impact of case volume on survival of septic shock in patients with malignancies*: Crit Care Med. 2012 Jan;40(1):55-62.

47. Azoulay É, Schlemmer B. Diagnostic strategy in cancer patients with acute respiratory failure. Intensive Care Med. 2006 Jun;32(6):808-22.

48. Azoulay E, Mokart D, Kouatchet A, Demoule A, Lemiale V. Acute respiratory failure in immunocompromised adults. Lancet Respir Med. 2019 Feb;7(2):173-86.

\section{Figures}




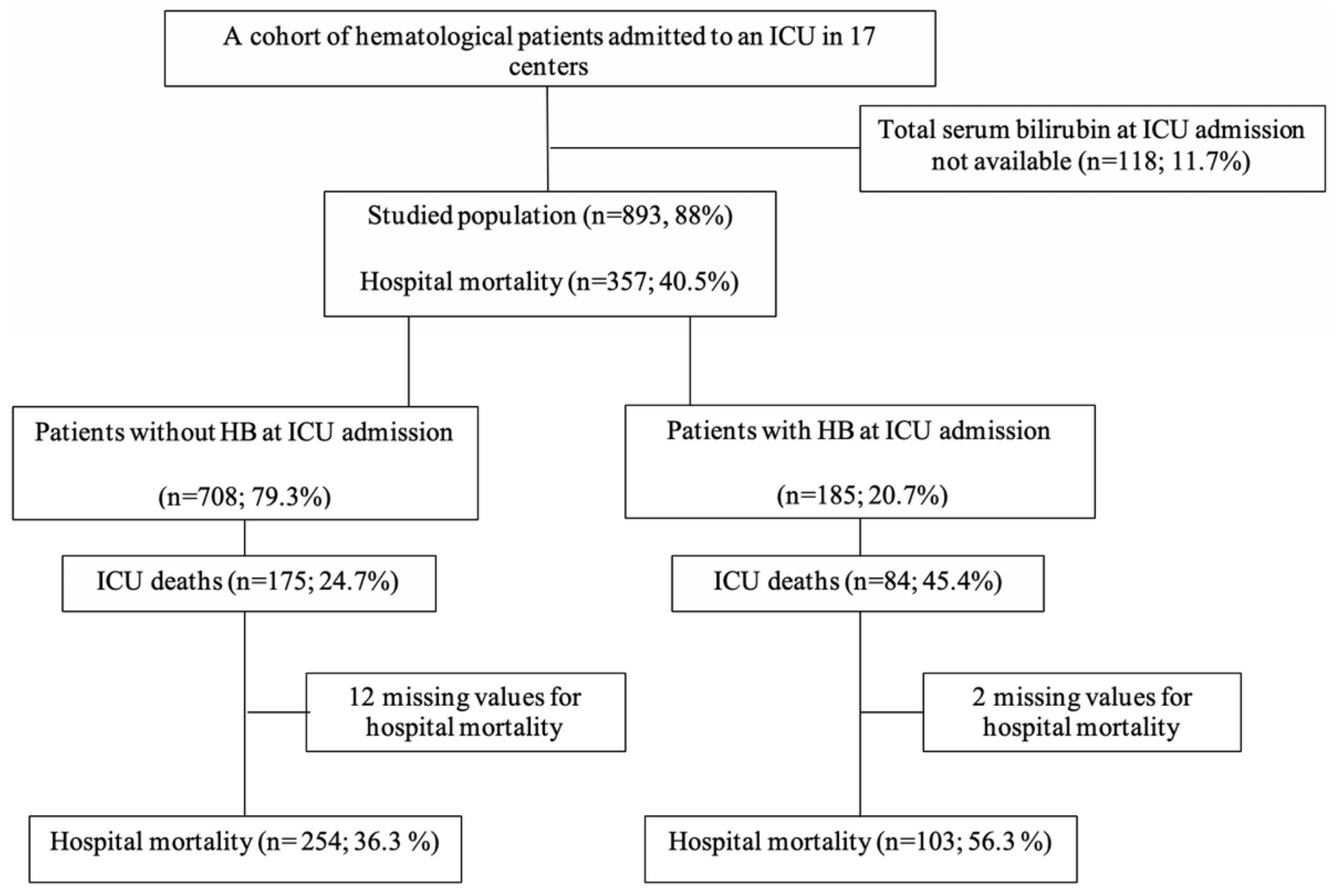

Figure 1

Flow chart of included patients. (ICU: Intensive care unit; HB: hyperbilirubinemia) 


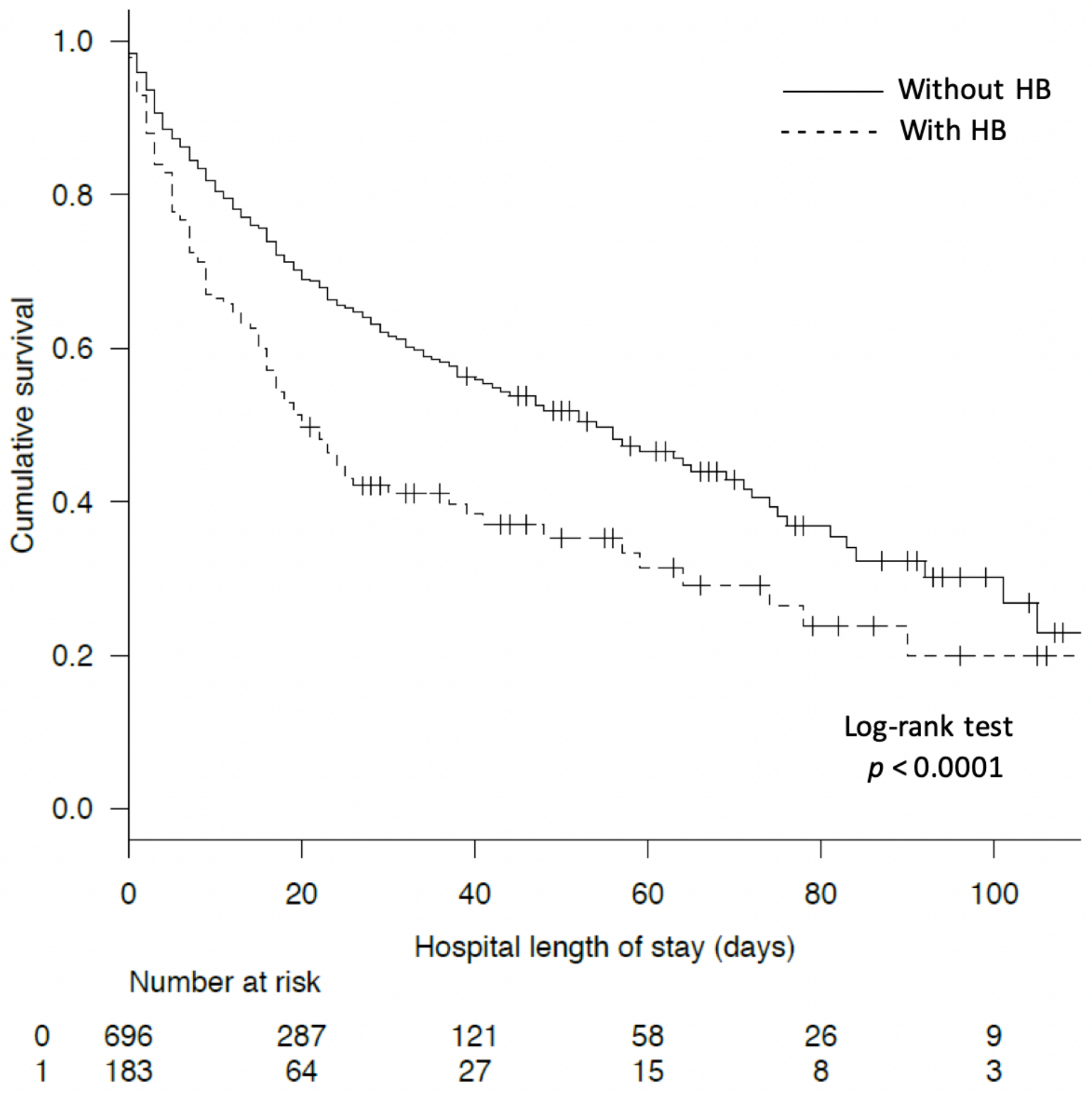

\section{Figure 2}

Hospital survival of critically ill hematological patients according to hyperbilirubinemia (total serum bilirubin $\geq 33 \mu \mathrm{mol} / \mathrm{L}$ ) at ICU admission. (ICU: Intensive care unit; HB: hyperbilirubinemia)

\section{Supplementary Files}

This is a list of supplementary files associated with this preprint. Click to download. 
- Additionaltable1.docx

Page 24/24 